\title{
Inequalities among Eigenvalues of Second-Order Difference Equations with General Coupled Boundary Conditions
}

\section{Chao Zhang and Shurong Sun}

\author{
School of Science, University of Jinan, Jinan, Shandong 250022, China \\ Correspondence should be addressed to Chao Zhang, ss_zhangc@ujn.edu.cn \\ Received 11 February 2009; Accepted 11 May 2009 \\ Recommended by Johnny Henderson \\ This paper studies general coupled boundary value problems for second-order difference \\ equations. Existence of eigenvalues is proved, numbers of their eigenvalues are calculated, \\ and their relationships between the eigenvalues of second-order difference equation with three \\ different coupled boundary conditions are established. \\ Copyright (C) 2009 C. Zhang and S. Sun. This is an open access article distributed under the \\ Creative Commons Attribution License, which permits unrestricted use, distribution, and \\ reproduction in any medium, provided the original work is properly cited.
}

\section{Introduction}

Consider the second-order difference equation

$$
-\nabla\left(p_{n} \Delta y_{n}\right)+q_{n} y_{n}=\lambda w_{n} y_{n}, \quad n \in[0, N-1]
$$

with the general coupled boundary condition

$$
\left(\begin{array}{c}
y_{N-1} \\
\Delta y_{N-1}
\end{array}\right)=e^{i \alpha} K\left(\begin{array}{c}
y_{-1} \\
\Delta y_{-1}
\end{array}\right),
$$

where $N \geq 2$ is an integer, $\Delta$ is the forward difference operator: $\Delta y_{n}=y_{n+1}-y_{n}, \nabla$ is the backward difference operator: $\nabla y_{n}=y_{n}-y_{n-1}$, and $p_{n}, q_{n}$, and $w_{n}$ are real numbers with $p_{n}>0$ for $n \in[-1, N-1], w_{n}>0$ for $n \in[0, N-1]$, and $p_{-1}=p_{N-1}=1 ; \lambda$ is the spectral 
parameter; the interval $[0, N-1]$ is the integral set $\{n\}_{n=0}^{N-1} ; \alpha,-\pi<\alpha \leq \pi$ is a constant parameter; $i=\sqrt{-1}$,

$$
K=\left(\begin{array}{ll}
k_{11} & k_{12} \\
k_{21} & k_{22}
\end{array}\right), \quad k_{i j} \in \mathbf{R}, \quad i, j=1,2, \text { with } \operatorname{det} K=1
$$

The boundary condition (1.2) contains the periodic and antiperiodic boundary conditions. In fact, (1.2) is the periodic boundary condition in the case where $\alpha=0$ and $K=I$, the identity matrix, and (1.2) is the antiperiodic condition in the case where $\alpha=\pi$ and $K=I$.

We first briefly recall some relative existing results of eigenvalue problems for difference equations. Atkinson [1, Chapter 6, Section 2] discussed the boundary conditions

$$
y_{-1}=\alpha y_{m-1}, \quad y_{m}=\beta y_{0}
$$

when he investigated the recurrence formula

$$
c_{n} y_{n+1}=\left(a_{n} \hat{\mathcal{\lambda}}+b_{n}\right) y_{n}-c_{n-1} y_{n-1}, \quad n \in[0, m-1],
$$

where $a_{n}, b_{n}, c_{n}, \alpha$, and $\beta$ are real numbers, subject to $a_{n}>0, c_{n}>0$, and

$$
\alpha c_{-1}=\beta c_{m-1}
$$

He remarked that all the eigenvalues of the boundary value problem (1.4) and (1.5) are real, and they may not be all distinct. If $c_{-1}=c_{m-1}$ and $\alpha=\beta=1$, he viewed the boundary conditions (1.4) as the periodic boundary conditions for (1.5). Shi and Chen [2] investigated the more general boundary value problem

$$
\begin{gathered}
-\nabla\left(C_{n} \Delta x_{n}\right)+B_{n} x_{n}=\lambda w_{n} x_{n}, \quad n \in[1, N], \quad N \geq 2, \\
R\left(\begin{array}{c}
-x_{0} \\
x_{N}
\end{array}\right)+S\left(\begin{array}{c}
C_{0} \Delta x_{0} \\
C_{N} \Delta x_{N}
\end{array}\right)=0,
\end{gathered}
$$

where $C_{n}, B_{n}$, and $w_{n}$ are $d \times d$ Hermitian matrices; $C_{0}$ and $C_{N}$ are nonsingular; $w_{n}>0$ for $n \in[1, N]$; $R$ and $S$ are $2 d \times 2 d$ matrices. Moreover, $R$ and $S$ satisfy $\operatorname{rank}(R, S)=2 d$ and the self-adjoint condition $R S^{*}=S R^{*}$ [2, Lemma 2.1]. A series of spectral results was obtained. We will remark that the boundary condition (1.8) includes the coupled boundary condition (1.2) when $d=1$, and the boundary conditions (1.4) when (1.6) holds. Agarwal and Wong studied existence of minimal and maximal quasisolutions of a second-order nonlinear periodic boundary value problem [3, Section 4]. In 2005, Wang and Shi [4] considered (1.1) with the periodic and antiperiodic boundary conditions. They found out the following results 
(see [4, Theorems 2.2 and 3.1]): the periodic and antiperiodic boundary value problems have exactly $N$ real eigenvalues $\left\{\lambda_{i}\right\}_{i=0}^{N-1}$ and $\left\{\tilde{\lambda}_{i}\right\}_{i=1}^{N}$, respectively, which satisfy

$$
\begin{aligned}
& \lambda_{0}<\tilde{\lambda}_{1} \leq \tilde{\lambda}_{2}<\lambda_{1} \leq \lambda_{2}<\tilde{\lambda}_{3} \leq \tilde{\lambda}_{4}<\cdots<\lambda_{N-2} \leq \lambda_{N-1}<\tilde{\lambda}_{N} \text {, if } N \text { is odd, } \\
& \lambda_{0}<\tilde{\lambda}_{1} \leq \tilde{\lambda}_{2}<\lambda_{1} \leq \lambda_{2}<\tilde{\lambda}_{3} \leq \tilde{\lambda}_{4}<\cdots<\tilde{\lambda}_{N-1} \leq \tilde{\lambda}_{N}<\lambda_{N-1} \text {, if } N \text { is even. }
\end{aligned}
$$

These results are similar to those about eigenvalues of periodic and antiperiodic boundary value problems for second-order ordinary differential equations (cf. [5-8]).

Motivated by [4], we compare the eigenvalues of the eigenvalue problem (1.1) with the coupled boundary condition (1.2) as $\alpha$ varies and obtain relationships between the eigenvalues in the present paper. These results extend the above results obtained in [4]. In this paper, we will apply some results obtained by Shi and Chen [2] to prove the existence of eigenvalues of (1.1) and (1.2) to calculate the number of these eigenvalues, and to apply some oscillation results obtained by Agarwal et al. [9] to compare the eigenvalues as $\alpha$ varies.

This paper is organized as follows. Section 2 gives some preliminaries including existence and numbers of eigenvalues of the coupled boundary value problems, and some properties of eigenvalues of a kind of separated boundary value problem, which will be used in the next section. Section 3 pays attention to comparison between the eigenvalues of problem (1.1) and (1.2) as $\alpha$ varies.

\section{Preliminaries}

Equation (1.1) can be rewritten as the recurrence formula

$$
p_{n} y_{n+1}=\left(p_{n}+p_{n-1}+q_{n}-\lambda w_{n}\right) y_{n}-p_{n-1} y_{n-1}, \quad n \in[0, N-1]
$$

Clearly, $y_{n}$ is a polynomial in $\lambda$ with real coefficients since $p_{n}, q_{n}$, and $w_{n}$ are all real. Hence, all the solutions of (1.1) are entire functions of $\lambda$. Especially, if $y_{0} \neq 0, y_{n}$ is a polynomial of degree $n$ in $\lambda$ for $n \leq N$. However, if $y_{-1} \neq 0$ and $y_{0}=0, y_{n}$ is a polynomial of degree $n-1$ in $\lambda$ for $n \leq N$.

We now prepare some results that are useful in the next section. The following lemma is mentioned in [4, Theorem 2.1].

Lemma 2.1 ([4, Theorem 2.1]). Let $y$ and $z$ be any solutions of (1.1). Then the Wronskian

$$
W[y, z](n)=\left|\begin{array}{cc}
y_{n+1} & z_{n+1} \\
p_{n} \Delta y_{n} & p_{n} \Delta z_{n}
\end{array}\right|=-p_{n}\left(y_{n+1} z_{n}-y_{n} z_{n+1}\right)
$$

is a constant on $[-1, N-1]$.

Theorem 2.2. If $k_{11} \neq k_{12}$ then the coupled boundary value problem (1.1) and (1.2) has exactly $N$ real eigenvalues. 
Proof. By setting $d=1, C_{n}=p_{n}, B_{n}=q_{n}$,

$$
R=\left(R_{1}, R_{2}\right)=\left(\begin{array}{ll}
e^{i \alpha} k_{11} & 1 \\
e^{i \alpha} k_{21} & 0
\end{array}\right), \quad S=\left(S_{1}, S_{2}\right)=\left(\begin{array}{ll}
-e^{i \alpha} k_{12} & 0 \\
-e^{i \alpha} k_{22} & 1
\end{array}\right),
$$

shifting the whole interval $[1, N]$ left by one unit, and using $p_{-1}=p_{N-1}=1,(1.1)$ and $(1.2)$ are written as (1.7) and (1.8), respectively. It is evident that $\operatorname{rank}(R, S)=2 d$ and $R S^{*}=S R^{*}$. Hence, the boundary condition (1.2) is self-adjoint by [2, Lemma 2.1]. In addition, it follows from (2.3) and $C_{-1}=1$ that

$$
\left(R_{1}+S_{1} C_{-1}, S_{2}\right)=\left(\begin{array}{cc}
e^{i \alpha}\left(k_{11}-k_{12}\right) & 0 \\
e^{i \alpha}\left(k_{21}-k_{22}\right) & 1
\end{array}\right)
$$

By noting that $k_{11} \neq k_{12}$, we get $\operatorname{rank}\left(R_{1}+S_{1} C_{-1}, S_{2}\right)=2$. Therefore, by [2, Theorem 4.1], the problem (1.1) and (1.2) has exactly $N$ real eigenvalues. This completes the proof.

Let $y_{n}(\lambda)$ be the solution of (1.1) with the initial conditions

$$
y_{-1}(\lambda)=0, \quad y_{0}(\lambda) \neq 0 .
$$

Consider the sequence

$$
y_{0}(\lambda), y_{1}(\lambda), \ldots, y_{N-1}(\lambda)
$$

If $y_{n}(\lambda)=0$ for some $n \in(0, N-1)$, then, we get from (2.1) that $y_{n-1}(\lambda)$ and $y_{n+1}(\lambda)$ have opposite signs. Hence, we say that sequence (2.6) exhibits a change of sign if $y_{n}(\lambda) y_{n+1}(\lambda)<0$ for some $n \in[0, N-1)$, or $y_{n}(\lambda)=0$ for some $n \in(0, N-1)$. A general zero of the sequence (2.6) is defined as its zero or a change of sign.

Now we consider (1.1) with the following separated boundary conditions:

$$
y_{-1}=0, \quad k_{12} \Delta y_{N-1}-k_{22} y_{N-1}=0,
$$

where $k_{12}, k_{22}$ are entries of $K$. It follows from (2.1) that the separated boundary value problem (1.1) with (2.7) has a unique solution, and the separated boundary value problem will be used to compare the eigenvalues of (1.1) and (1.2) as $\alpha$ varies in the next section.

In [9], Agarwal et al. studied the following boundary value problem on time scales:

$$
y^{\Delta \Delta}+q(t) y^{\sigma}=-\lambda y^{\sigma}, \quad t \in[\rho(a), \rho(b)] \cap \mathbf{T},
$$

with the boundary conditions

$$
R_{a}(y):=\alpha y(\rho(a))+\beta y^{\Delta}(\rho(a))=0, \quad R_{b}(y):=\gamma y(b)+\delta y^{\Delta}(b)=0,
$$


where $\mathbf{T}$ is a time scale, $\sigma(t)$ and $\rho(t)$ are the forward and backward jump operators in $\mathbf{T}$, $y^{\Delta}$ is the delta derivative, and $y^{\sigma}(t):=y(\sigma(t)) ; q:[\rho(a), \rho(b)] \cap \mathbf{T} \rightarrow \mathbf{R}$ is continuous; $\left(\alpha^{2}+\beta^{2}\right)\left(\gamma^{2}+\delta^{2}\right) \neq 0 ; a, b \in \mathrm{T}$ with $a<b$. They obtained some useful oscillation results. With a similar argument to that used in the proof of [9, Theorem 1], one can show the following result.

Lemma 2.3. The eigenvalues of the boundary value problem are

$$
-\left(p(t) y^{\Delta}(t)\right)^{\Delta}+q^{\sigma}(t) y^{\sigma}(t)=\lambda r^{\sigma}(t) y^{\sigma}(t), \quad t \in[\rho(a), \rho(b)] \cap \mathbf{T},
$$

with

$$
R_{a}(y)=R_{b}(y)=0
$$

where $p^{\Delta}, q^{\sigma}$, and $r^{\sigma}$ are real and continuous functions in $[\rho(a), \rho(b)] \cap \mathbf{T}, p>0$ over $[\rho(a), b] \cap$ $\mathbf{T}, r^{\sigma}>0$ over $[\rho(a), \rho(b)] \cap \mathbf{T}, p(\rho(a))=p(b)=1$ are arranged as $-\infty<\lambda_{0}<\lambda_{1}<\lambda_{2}<\cdots$, and an eigenfunction corresponding to $\lambda_{k}$ has exactly $k$ generalized zeros in the open interval $(a, b)$.

By setting $[\rho(a), b] \cap \mathbf{T}=[-1, N-1]:=\{n\}_{-1}^{N-1}, \alpha=1, \beta=0, \gamma=-k_{22}, \delta=k_{12}$, the above boundary value problem can be written as (1.1) with (2.7), then we have the following result.

Lemma 2.4. The boundary value problem (1.1) and (2.7) has $N-1$ real and simple eigenvalues as $k_{12}=0$ and $N$ real and simple eigenvalues as $k_{12} \neq 0$, which can be arranged in the increasing order

$$
\mu_{0}<\mu_{1}<\cdots<\mu_{N_{s}} \text {, where } N_{s}:=N-2 \text { or } N-1 \text {. }
$$

Let $y_{n}(\lambda)$ be the solution of (1.1) with the separated boundary conditions (2.7). Then sequence (2.6) exhibits no changes of sign for $\lambda \leq \mu_{0}$, exactly $k+1$ changes of sign for $\mu_{k}<\lambda \leq \mu_{k+1}\left(0 \leq k \leq N_{s}-1\right)$, and $N_{s}+1$ changes of sign for $\lambda>\mu_{N_{s}}$.

Let $\varphi_{n}$ and $\psi_{n}$ be the solutions of (1.1) satisfying the following initial conditions:

$$
\varphi_{-1}=\psi_{0}=1, \quad \varphi_{0}=\psi_{-1}=0,
$$

respectively. By Lemma 2.1 and using $p_{N-1}=1$, we have

$$
\Delta \varphi_{N-1} \psi_{N-1}-\varphi_{N-1} \Delta \psi_{N-1}=\varphi_{N} \psi_{N-1}-\varphi_{N-1} \psi_{N}=-1
$$

Obviously, $\varphi_{n}(\lambda)$ and $\psi_{n}(\lambda)$ are two linearly independent solutions of (1.1). The following lemma can be derived from [4, Proposition 3.1]. 
Lemma 2.5. Let $\mu_{k}\left(0 \leq k \leq N_{s}\right)$ be the eigenvalues of (1.1) and (2.7) with $k_{12}=0$ and be arranged as (2.12). Then, $\psi_{n}\left(\mu_{k}\right)$ is an eigenfunction of the problem (1.1) and (2.7) with respect to $\mu_{k}(0 \leq k \leq$ $\left.N_{s}\right)$, that is, for $0 \leq k \leq N_{s}, \psi_{n}\left(\mu_{k}\right)$ is a nontrivial solution of (1.1) satisfying

$$
\psi_{-1}\left(\mu_{k}\right)=\psi_{N-1}\left(\mu_{k}\right)=0
$$

Moreover, if $k$ is odd, $\psi_{N}\left(\mu_{k}\right)>0$ and if $k$ is even, $\psi_{N}\left(\mu_{k}\right)<0$ for $2 \leq k \leq N_{s}$.

A representation of solutions for a nonhomogeneous linear equation with initial conditions is given by the following lemma.

Lemma 2.6 (see [4, Theorem 2.3]). For any $\left\{f_{n}\right\}_{n=0}^{N-1} \subset \mathbf{C}$ and for any $c_{-1}, c_{0} \in \mathbf{C}$, the initial value problem

$$
\begin{gathered}
-\nabla\left(p_{n} \Delta z_{n}\right)+\left(q_{n}-\lambda w_{n}\right) z_{n}=w_{n} f_{n}, \quad n \in[0, N-1] \\
z_{-1}=c_{-1}, \quad z_{0}=c_{0}
\end{gathered}
$$

has a unique solution $z$, which can be expressed as

$$
z_{n}=c_{-1} \varphi_{n}+c_{0} \psi_{n}+\sum_{j=0}^{n-1} w_{j}\left(\varphi_{n} \psi_{j}-\varphi_{j} \psi_{n}\right) f_{j}, \quad n \in[-1, N]
$$

where $\sum_{j=0}^{-2} \cdot=\sum_{j=0}^{-1} \cdot:=0$.

\section{Main Results}

Let $\varphi_{n}$ and $\psi_{n}$ be defined in Section 2, let $\mu_{k}\left(0 \leq k \leq N_{s}\right)$ be the eigenvalues of the separated boundary value problem (1.1) with (2.7), and let $\lambda_{j}\left(e^{i \alpha} K\right)(0 \leq j \leq N-1)$ be the eigenvalues of the coupled boundary value problem (1.1) and (1.2) and arranged in the nondecreasing order

$$
\lambda_{0}\left(e^{i \alpha} K\right) \leq \lambda_{1}\left(e^{i \alpha} K\right) \leq \cdots \leq \lambda_{N-1}\left(e^{i \alpha} K\right)
$$

Clearly, $\lambda_{j}(K) \quad(0 \leq j \leq N-1)$ denotes the eigenvalue of the problem (1.1) and (1.2) with $\alpha=0$, and $\lambda_{j}(-K) \quad(0 \leq j \leq N-1)$ denotes the eigenvalue of the problem (1.1) and (1.2) with $\alpha=\pi$. We now present the main results of this paper. 
Theorem 3.1. Assume that $k_{11}>0, k_{12} \leq 0$ or $k_{11} \geq 0, k_{12}<0$. Then, for every fixed $\alpha \neq 0$, $-\pi<\alpha<\pi$, one has the following inequalities:

$$
\begin{aligned}
\lambda_{0}(K)<\lambda_{0}\left(e^{i \alpha} K\right)<\lambda_{0}(-K) & \leq \lambda_{1}(-K)<\lambda_{1}\left(e^{i \alpha} K\right)<\lambda_{1}(K) \\
& \leq \lambda_{2}(K)<\lambda_{2}\left(e^{i \alpha} K\right)<\lambda_{2}(-K) \\
& \leq \lambda_{3}(-K)<\lambda_{3}\left(e^{i \alpha} K\right)<\lambda_{3}(K) \\
& \leq \cdots \leq \lambda_{N-2}(-K)<\lambda_{N-2}\left(e^{i \alpha} K\right)<\lambda_{N-2}(K) \\
& \leq \lambda_{N-1}(K)<\lambda_{N-1}\left(e^{i \alpha} K\right)<\lambda_{N-1}(-K), \quad \text { if } N \text { is odd, } \\
\lambda_{0}(K)<\lambda_{0}\left(e^{i \alpha} K\right)<\lambda_{0}(-K) & \leq \lambda_{1}(-K)<\lambda_{1}\left(e^{i \alpha} K\right)<\lambda_{1}(K) \\
& \leq \lambda_{2}(K)<\lambda_{2}\left(e^{i \alpha} K\right)<\lambda_{2}(-K) \\
& \leq \lambda_{3}(-K)<\lambda_{3}\left(e^{i \alpha} K\right)<\lambda_{3}(K) \\
& \leq \cdots \leq \lambda_{N-2}(K)<\lambda_{N-2}\left(e^{i \alpha} K\right)<\lambda_{N-2}(-K) \\
& \leq \lambda_{N-1}(-K)<\lambda_{N-1}\left(e^{i \alpha} K\right)<\lambda_{N-1}(K), \quad \text { if } N \text { is even. }
\end{aligned}
$$

Remark 3.2. If $k_{11} \leq 0, k_{12}>0$ or $k_{11}<0, k_{12} \geq 0$, a similar result can be obtained by applying Theorem 3.1 to $-K$. In fact, $e^{i \alpha} K=e^{i(\pi+\alpha)}(-K)$ for $\alpha \in(-\pi, 0)$ and $e^{i \alpha} K=e^{i(-\pi+\alpha)}(-K)$ for $\alpha \in$ $(0, \pi)$. Hence, the boundary condition (1.2) in the cases of $k_{11} \leq 0, k_{12}>0$ or $k_{11}<0, k_{12} \geq 0$ and $\alpha \neq 0,-\pi<\alpha<\pi$, can be written as condition (1.2), where $\alpha$ is replaced by $\pi+\alpha$ for $\alpha \in(-\pi, 0)$ and $-\pi+\alpha$ for $\alpha \in(0, \pi)$, and $K$ is replaced by $-K$.

Before proving Theorem 3.1, we prove the following five propositions.

Proposition 3.3. For $\lambda \in \mathrm{C}, \lambda$ is an eigenvalue of (1.1) and (1.2) if and only if

$$
f(\lambda)=2 \cos \alpha,
$$

where

$$
f(\lambda):=k_{22} \varphi_{N-1}(\lambda)+\left(k_{11}-k_{12}\right) \Delta \psi_{N-1}(\lambda)-\left(k_{21}-k_{22}\right) \psi_{N-1}(\lambda)-k_{12} \Delta \varphi_{N-1}(\lambda) .
$$

Moreover, $\lambda$ is a multiple eigenvalue of (1.1) and (1.2) if and only if

$$
\begin{array}{cc}
\varphi_{N-1}(\lambda)=e^{i \alpha}\left(k_{11}-k_{12}\right), \quad \Delta \varphi_{N-1}(\lambda)=e^{i \alpha}\left(k_{21}-k_{22}\right), \\
\psi_{N-1}(\lambda)=e^{i \alpha} k_{12}, \quad \Delta \psi_{N-1}(\lambda)=e^{i \alpha} k_{22} .
\end{array}
$$


Proof. Since $\varphi_{n}$ and $\psi_{n}$ are linearly independent solutions of (1.1), then $\lambda$ is an eigenvalue of the problem (1.1) and (1.2) if and only if there exist two constants $C_{1}$ and $C_{2}$ not both zero such that $C_{1} \varphi_{n}+C_{2} \psi_{n}$ satisfies (1.2), which yields

$$
\left(\begin{array}{cc}
\varphi_{N-1}(\lambda)-e^{i \alpha}\left(k_{11}-k_{12}\right) & \psi_{N-1}(\lambda)-e^{i \alpha} k_{12} \\
\Delta \varphi_{N-1}(\lambda)-e^{i \alpha}\left(k_{21}-k_{22}\right) & \Delta \psi_{N-1}(\lambda)-e^{i \alpha} k_{22}
\end{array}\right)\left(\begin{array}{c}
C_{1} \\
C_{2}
\end{array}\right)=0
$$

It is evident that (3.6) has a nontrivial solution $\left(C_{1}, C_{2}\right)$ if and only if

$$
\operatorname{det}\left(\begin{array}{cc}
\varphi_{N-1}(\lambda)-e^{i \alpha}\left(k_{11}-k_{12}\right) & \psi_{N-1}(\lambda)-e^{i \alpha} k_{12} \\
\Delta \varphi_{N-1}(\lambda)-e^{i \alpha}\left(k_{21}-k_{22}\right) & \Delta \psi_{N-1}(\lambda)-e^{i \alpha} k_{22}
\end{array}\right)=0
$$

which, together with (2.14) and det $K=1$, implies that

$$
1+e^{2 i \alpha}-e^{i \alpha} f(\lambda)=0
$$

Then (3.3) follows from the above relation and the fact that $e^{-i \alpha}+e^{i \alpha}=2 \cos \alpha$. On the other hand, (1.1) has two linearly independent solutions satisfying (1.2) if and only if all the entries of the coefficient matrix of (3.6) are zero. Hence, $\lambda$ is a multiple eigenvalue of (1.1) and (1.2) if and only if (3.5) holds. This completes the proof.

The following result is a direct consequence of the first result of Proposition 3.3.

Corollary 3.4. For any $\alpha \in(-\pi, \pi]$,

$$
\lambda_{j}\left(e^{i \alpha} K\right)=\lambda_{j}\left(e^{-i \alpha} K\right), \quad 0 \leq j \leq N-1
$$

Proposition 3.5. Assume that $k_{11}>0, k_{12} \leq 0$ or $k_{11} \geq 0, k_{12}<0$. Then one has the following results.

(i) For each $k, 0 \leq k \leq N_{s}, f\left(\mu_{k}\right) \geq 2$ if $k$ is odd, and $f\left(\mu_{k}\right) \leq-2$ if $k$ is even.

(ii) There exists a constant $v_{0}<\mu_{0}$ such that $f\left(v_{0}\right) \geq 2$.

(iii) If the boundary value problem (1.1) and (2.7) has exactly $N-1$ eigenvalues then there exists a constant $\xi_{0}$ such that $\mu_{N-2}<\xi_{0}$ and $f\left(\xi_{0}\right) \leq-2$, where $N$ is odd, and there exists a constant $\eta_{0}$ such that $\mu_{N-2}<\eta_{0}$ and $f\left(\eta_{0}\right) \geq 2$, where $N$ is even.

Proof. (i) If $\psi_{n}\left(\mu_{k}\right)$ is an eigenfunction of the problem (1.1) and (2.7) respect to $\mu_{k}$ then $k_{12} \Delta \psi_{N-1}\left(\mu_{k}\right)-k_{22} \psi_{N-1}\left(\mu_{k}\right)=0$. By Lemma 2.3 and the initial conditions (2.13), we have that if $k_{12}<0$ then the sequence $\psi_{0}\left(\mu_{k}\right), \psi_{1}\left(\mu_{k}\right), \ldots, \psi_{N-1}\left(\mu_{k}\right)$ exhibits $k$ changes of sign and

$$
\operatorname{sgn} \psi_{N-1}\left(\mu_{k}\right)=(-1)^{k}
$$


Case 1. If $k_{12}<0$ then it follows from $k_{12} \Delta \psi_{N-1}\left(\mu_{k}\right)-k_{22} \psi_{N-1}\left(\mu_{k}\right)=0$ that

$$
\frac{\psi_{N-1}\left(\mu_{k}\right)}{k_{12}}=\frac{\Delta \psi_{N-1}\left(\mu_{k}\right)}{k_{22}}, \quad k_{11} k_{22} \psi_{N-1}\left(\mu_{k}\right)=k_{11} k_{12} \Delta \psi_{N-1}\left(\mu_{k}\right)
$$

By (2.14) and the first relation in (3.11), for each $k, 0 \leq k \leq N_{s}$, we have

$$
\begin{aligned}
\varphi_{N-1}\left(\mu_{k}\right) \Delta \psi_{N-1}\left(\mu_{k}\right)-\Delta \varphi_{N-1}\left(\mu_{k}\right) \psi_{N-1}\left(\mu_{k}\right) \\
=\varphi_{N-1}\left(\mu_{k}\right) \frac{k_{22}}{k_{12}} \psi_{N-1}\left(\mu_{k}\right)-\Delta \varphi_{N-1}\left(\mu_{k}\right) \psi_{N-1}\left(\mu_{k}\right) \\
=\left(k_{22} \varphi_{N-1}\left(\mu_{k}\right)-k_{12} \Delta \varphi_{N-1}\left(\mu_{k}\right)\right) \frac{\psi_{N-1}\left(\mu_{k}\right)}{k_{12}}=1 .
\end{aligned}
$$

By the definition of $f(\lambda),(3.11)$, and $\operatorname{det} K=1$,

$$
\begin{aligned}
k_{12} f\left(\mu_{k}\right)= & k_{12} k_{22} \varphi_{N-1}\left(\mu_{k}\right)+k_{12}\left(k_{11}-k_{12}\right) \Delta \psi_{N-1}\left(\mu_{k}\right) \\
& -k_{12}\left(k_{21}-k_{22}\right) \psi_{N-1}\left(\mu_{k}\right)-k_{12}^{2} \Delta \varphi_{N-1}\left(\mu_{k}\right) \\
= & k_{12} k_{22} \varphi_{N-1}\left(\mu_{k}\right)+k_{11} k_{12} \Delta \psi_{N-1}\left(\mu_{k}\right)-k_{12} k_{21} \psi_{N-1}\left(\mu_{k}\right)-k_{12}^{2} \Delta \varphi_{N-1}\left(\mu_{k}\right) \\
= & k_{12} k_{22} \varphi_{N-1}\left(\mu_{k}\right)+k_{11} k_{22} \psi_{N-1}\left(\mu_{k}\right)-k_{12} k_{21} \psi_{N-1}\left(\mu_{k}\right)-k_{12}^{2} \Delta \varphi_{N-1}\left(\mu_{k}\right) \\
= & k_{12} k_{22} \varphi_{N-1}\left(\mu_{k}\right)-k_{12}^{2} \Delta \varphi_{N-1}\left(\mu_{k}\right)+\psi_{N-1}\left(\mu_{k}\right) .
\end{aligned}
$$

Hence,

$$
f\left(\mu_{k}\right)=\left(k_{22} \varphi_{N-1}\left(\mu_{k}\right)-k_{12} \Delta \varphi_{N-1}\left(\mu_{k}\right)\right)+\frac{\psi_{N-1}\left(\mu_{k}\right)}{k_{12}} .
$$

Noting $\left(k_{22} \varphi_{N-1}\left(\mu_{k}\right)-k_{12} \Delta \varphi_{N-1}\left(\mu_{k}\right)\right)\left(\psi_{N-1}\left(\mu_{k}\right) / k_{12}\right)=1, k_{12}<0$, and (3.10), we have that if $k$ is odd then

$$
f\left(\mu_{k}\right)=\left(\sqrt{\frac{\psi_{N-1}\left(\mu_{k}\right)}{k_{12}}}-\sqrt{k_{22} \varphi_{N-1}\left(\mu_{k}\right)-k_{12} \Delta \varphi_{N-1}\left(\mu_{k}\right)}\right)^{2}+2 \geq 2,
$$

and if $k$ is even then

$$
f\left(\mu_{k}\right)=-\left(\sqrt{-\frac{\psi_{N-1}\left(\mu_{k}\right)}{k_{12}}}-\sqrt{-\left(k_{22} \varphi_{N-1}\left(\mu_{k}\right)-k_{12} \Delta \varphi_{N-1}\left(\mu_{k}\right)\right)}\right)^{2}-2 \leq-2 .
$$


Case 2. If $k_{12}=0$ then it follows from (2.7) and (2.14) that for each $k, 0 \leq k \leq N_{s}$,

$$
\varphi_{N-1}\left(\mu_{k}\right) \psi_{N}\left(\mu_{k}\right)=1
$$

From (2.15) and by the definition of $f(\lambda)$, we get

$$
f\left(\mu_{k}\right)=\frac{k_{22}}{\psi_{N}\left(\mu_{k}\right)}+k_{11} \psi_{N}\left(\mu_{k}\right)
$$

Hence, noting det $K=k_{11} k_{22}=1, k_{11}>0$, and by Lemma 2.5 , we have that if $k$ is odd, then

$$
f\left(\mu_{k}\right) \geq 2,
$$

and if $k$ is even, then

$$
f\left(\mu_{k}\right) \leq-2
$$

(ii) By the discussions in the first paragraph of Section $2, \varphi_{N-1}(\lambda)$ is a polynomial of degree $N-2$ in $\lambda, \varphi_{N}(\lambda)$ is a polynomial of degree $N-1$ in $\lambda, \psi_{N-1}(\lambda)$ is a polynomial of degree $N-1$ in $\lambda$, and $\psi_{N}(\lambda)$ is a polynomial of degree $N$ in $\lambda$. Further, $\psi_{N}(\lambda)$ can be written as

$$
\psi_{N}(\lambda)=(-1)^{N} A_{N} \lambda^{N}+A_{N-1} \lambda^{N-1}+\cdots+A_{0}
$$

where $A_{N}=w_{0} w_{1} \cdots w_{N-1}\left(p_{0} p_{1} \cdots p_{N-1}\right)^{-1}>0$ and $A_{n}$ is a certain constant for $n \in[0, N-1]$. Then

$$
f(\lambda)=(-1)^{N}\left(k_{11}-k_{12}\right) A_{N} \lambda^{N}+h(\lambda),
$$

where $h(\lambda)$ is a polynomial in $\lambda$ whose degree is not larger than $N-1$. Clearly, as $\lambda \rightarrow-\infty$, $f(\lambda) \rightarrow+\infty$ since $\left(k_{11}-k_{12}\right)>0$. By the first part of this proposition, $f\left(\mu_{0}\right) \leq-2$. So there exists a constant $v_{0}<\mu_{0}$ such that $f\left(v_{0}\right) \geq 2$.

(iii) It follows from the first part of this proposition that if $N$ is odd, $f\left(\mu_{N-2}\right) \geq 2$ and if $N$ is even, $f\left(\mu_{N-2}\right) \leq-2$. By (3.22), if $N$ is odd, $f(\lambda) \rightarrow-\infty$ as $\lambda \rightarrow+\infty$; if $N$ is even, $f(\lambda) \rightarrow+\infty$ as $\lambda \rightarrow+\infty$. Hence, if $N$ is odd, there exists a constant $\xi_{0}>\mu_{N-2}$ such that $f\left(\xi_{0}\right) \leq-2$; if $N$ is even, there exists a constant $\eta_{0}>\mu_{N-2}$ such that $f\left(\eta_{0}\right) \geq 2$. This completes the proof.

Since $\varphi_{n}$ and $\psi_{n}$ are both polynomials in $\lambda$, so is $f(\lambda)$. Denote

$$
\frac{d}{d \lambda} f(\lambda):=f^{\prime}(\lambda), \quad \frac{d^{2}}{d \lambda^{2}} f(\lambda):=f^{\prime \prime}(\lambda)
$$


Proposition 3.6. Assume that $k_{11}>0, k_{12} \leq 0$ or $k_{11} \geq 0, k_{12}<0$. Equations $f^{\prime}(\lambda)=0$ and $f(\lambda)=2$ or -2 hold if and only if $\lambda$ is a multiple eigenvalue of (1.1) and (1.2) with $\alpha=0$ or $\alpha=\pi$. If $f(\lambda)=2$ or -2 for some $\lambda \neq \mu_{k}\left(0 \leq k \leq N_{s}\right)$, then $\lambda$ is a simple eigenvalue of (1.1) and (1.2) with $\alpha=0$ or $\alpha=\pi$ and for every $\lambda \neq \mu_{k}\left(0 \leq k \leq N_{s}\right)$, with $-2 \leq f(\lambda) \leq 2$ one has:

$$
\begin{gathered}
f^{\prime}(\lambda)<0, \quad \lambda<\mu_{0}, \\
(-1)^{k} f^{\prime}(\lambda)>0, \quad \mu_{k}<\lambda<\mu_{k+1}, \quad 0 \leq k \leq N-3, \\
(-1)^{N-2} f^{\prime}(\lambda)>0, \quad \lambda>\mu_{N-2} .
\end{gathered}
$$

Proof. Since $\varphi_{n}$ and $\psi_{n}$ are solutions of (1.1), we have

$$
\begin{aligned}
& -\nabla\left(p_{n} \Delta \varphi_{n}(\lambda)\right)+q_{n} \varphi_{n}(\lambda)=\lambda w_{n} \varphi_{n}(\lambda), \\
& -\nabla\left(p_{n} \Delta \psi_{n}(\lambda)\right)+q_{n} \psi_{n}(\lambda)=\lambda w_{n} \psi_{n}(\lambda) .
\end{aligned}
$$

Differentiating (3.25) and (3.26) with respect to $\lambda$, respectively, yields that

$$
\begin{aligned}
& -\nabla\left(p_{n} \Delta \varphi_{n}^{\prime}(\lambda)\right)+\left(q_{n}-\lambda w_{n}\right) \varphi_{n}^{\prime}(\lambda)=w_{n} \varphi_{n}(\lambda), \\
& -\nabla\left(p_{n} \Delta \psi_{n}^{\prime}(\lambda)\right)+\left(q_{n}-\lambda w_{n}\right) \psi_{n}^{\prime}(\lambda)=w_{n} \psi_{n}(\lambda) .
\end{aligned}
$$

It follows from (2.13) that

$$
\varphi_{0}^{\prime}=\varphi_{-1}^{\prime}=\psi_{0}^{\prime}=\psi_{-1}^{\prime}=0
$$

Thus, by Lemma 2.6 and from (3.27)-(3.28), we have

$$
\begin{aligned}
\varphi_{n}^{\prime}(\lambda) & =\sum_{j=0}^{n-1} w_{j} \varphi_{j}(\lambda)\left(\varphi_{n}(\lambda) \psi_{j}(\lambda)-\varphi_{j}(\lambda) \psi_{n}(\lambda)\right), \\
\psi_{n}^{\prime}(\lambda) & =\sum_{j=0}^{n-1} w_{j} \psi_{j}(\lambda)\left(\varphi_{n}(\lambda) \psi_{j}(\lambda)-\varphi_{j}(\lambda) \psi_{n}(\lambda)\right) .
\end{aligned}
$$

It follows from (3.29) that

$$
\begin{aligned}
\Delta \varphi_{n-1}^{\prime}(\lambda) & =\sum_{j=0}^{n-1} w_{j} \varphi_{j}(\lambda)\left(\Delta \varphi_{n-1}(\lambda) \psi_{j}(\lambda)-\varphi_{j}(\lambda) \Delta \psi_{n-1}(\lambda)\right), \\
\Delta \psi_{n-1}^{\prime}(\lambda) & =\sum_{j=0}^{n-1} w_{j} \psi_{j}(\lambda)\left(\Delta \varphi_{n-1}(\lambda) \psi_{j}(\lambda)-\varphi_{j}(\lambda) \Delta \psi_{n-1}(\lambda)\right) .
\end{aligned}
$$


Hence, not indicating $\lambda$ explicitly, we get

$$
\begin{aligned}
f^{\prime}= & k_{22} \varphi_{N-1}^{\prime}+\left(k_{11}-k_{12}\right) \Delta \psi_{N-1}^{\prime}-\left(k_{21}-k_{22}\right) \psi_{N-1}^{\prime}-k_{12} \Delta \varphi_{N-1}^{\prime} \\
= & k_{22} \sum_{j=0}^{N-2} w_{j} \varphi_{j}\left(\varphi_{N-1} \psi_{j}-\varphi_{j} \psi_{N-1}\right)+\left(k_{11}-k_{12}\right) \sum_{j=0}^{N-1} w_{j} \psi_{j}\left(\Delta \varphi_{N-1} \psi_{j}-\varphi_{j} \Delta \psi_{N-1}\right) \\
& -\left(k_{21}-k_{22}\right) \sum_{j=0}^{N-2} w_{j} \psi_{j}\left(\varphi_{N-1} \psi_{j}-\varphi_{j} \psi_{N-1}\right)-k_{12} \sum_{j=0}^{N-1} w_{j} \varphi_{j}\left(\Delta \varphi_{N-1} \psi_{j}-\varphi_{j} \Delta \psi_{N-1}\right) \\
= & \sum_{j=0}^{N-1} w_{j} \delta_{j}
\end{aligned}
$$

where

$$
\begin{aligned}
& \delta_{j}:=\left(\left(k_{11}-k_{12}\right) \Delta \varphi_{N-1}-\left(k_{21}-k_{22}\right) \varphi_{N-1}\right) \psi_{j}^{2} \\
&+\left(k_{22} \varphi_{N-1}-\left(k_{11}-k_{12}\right) \Delta \psi_{N-1}+\left(k_{21}-k_{22}\right) \psi_{N-1}-k_{12} \Delta \varphi_{N-1}\right) \psi_{j} \varphi_{j} \\
&+\left(k_{12} \Delta \psi_{N-1}-k_{22} \psi_{N-1}\right) \varphi_{j}^{2} \\
&=\left(\psi_{j}, \varphi_{j}\right) I\left(\begin{array}{c}
\psi_{j} \\
\varphi_{j}
\end{array}\right), \\
& I:=\left(\begin{array}{cc}
\left(k_{11}-k_{12}\right) \Delta \varphi_{N-1}-\left(k_{21}-k_{22}\right) \varphi_{N-1} & \frac{1}{2}\left(k_{22} \varphi_{N-1}-\left(k_{11}-k_{12}\right) \Delta \psi_{N-1}\right. \\
\frac{1}{2}\left(k_{22} \varphi_{N-1}-\left(k_{11}-k_{12}\right) \Delta \psi_{N-1}\right. & \left.+\left(k_{21}-k_{22}\right) \psi_{N-1}-k_{12} \Delta \varphi_{N-1}\right) \\
\left.+\left(k_{21}-k_{22}\right) \psi_{N-1}-k_{12} \Delta \varphi_{N-1}\right) & k_{12} \Delta \psi_{N-1}-k_{22} \psi_{N-1}
\end{array}\right),
\end{aligned}
$$

which is symmetric for any $\lambda \in \mathbf{R}$. Then, we have

$$
\begin{aligned}
\operatorname{det} I(\lambda)= & \left(k_{12} \Delta \psi_{N-1}(\lambda)-k_{22} \psi_{N-1}(\lambda)\right)\left(\left(k_{11}-k_{12}\right) \Delta \varphi_{N-1}(\lambda)-\left(k_{21}-k_{22}\right) \varphi_{N-1}(\lambda)\right) \\
& -\frac{\left(k_{22} \varphi_{N-1}(\lambda)-\left(k_{11}-k_{12}\right) \Delta \psi_{N-1}(\lambda)+\left(k_{21}-k_{22}\right) \psi_{N-1}(\lambda)-k_{12} \Delta \varphi_{N-1}(\lambda)\right)^{2}}{4} \\
= & -\frac{1}{4} f^{2}(\lambda)+1
\end{aligned}
$$

Hence, if $f(\lambda)=2$ or -2 , we get from (3.33) that det $I(\lambda)=0$. Then, for any fixed $\lambda$ with $f(\lambda)=2$ or -2 , the matrix $I(\lambda)$ is positive semidefinite or negative semidefinite. Therefore, for such a $\lambda, f_{\prime}(\lambda)$ cannot vanish unless $\delta_{j}(\lambda)=0$ for all $0 \leq j \leq N-1$. Because $\varphi_{n}$ and $\psi_{n}$ are 
linearly independent, $\delta_{j}(\lambda)$ is identically zero if and only if all the entries of the matrix $I(\lambda)$ vanish, namely,

$$
\begin{gathered}
k_{12} \Delta \psi_{N-1}(\lambda)-k_{22} \psi_{N-1}(\lambda)=0, \\
\left(k_{11}-k_{12}\right) \Delta \varphi_{N-1}(\lambda)-\left(k_{21}-k_{22}\right) \varphi_{N-1}(\lambda)=0, \\
k_{22} \varphi_{N-1}(\lambda)-\left(k_{11}-k_{12}\right) \Delta \psi_{N-1}(\lambda)+\left(k_{21}-k_{22}\right) \psi_{N-1}(\lambda)-k_{12} \Delta \varphi_{N-1}(\lambda)=0
\end{gathered}
$$

which, together with $f(\lambda)=2$ and $\operatorname{det} K=1$, implies

$$
\begin{array}{cl}
\varphi_{N-1}(\lambda)=k_{11}-k_{12}, & \Delta \varphi_{N-1}(\lambda)=k_{21}-k_{22}, \\
\psi_{N-1}(\lambda)=k_{12}, & \Delta \psi_{N-1}(\lambda)=k_{22} .
\end{array}
$$

Then by Proposition 3.3, $\lambda$ is a multiple eigenvalue of (1.1) and (1.2) with $\alpha=0$. In addition, (3.34), together with $f(\lambda)=-2$ and det $K=1$, implies

$$
\begin{array}{cl}
\varphi_{N-1}(\lambda)=-\left(k_{11}-k_{12}\right), \quad & \Delta \varphi_{N-1}(\lambda)=-\left(k_{21}-k_{22}\right), \\
\psi_{N-1}(\lambda)=-k_{12}, & \Delta \psi_{N-1}(\lambda)=-k_{22} .
\end{array}
$$

Then by Proposition 3.3, $\lambda$ is a multiple eigenvalue of (1.1) and (1.2) with $\alpha=\pi$. Conversely, from (3.35) or (3.36), it can be easily verified that (3.34) holds, then $f^{\prime}(\lambda)=0$. It follows again from (3.35) or (3.36) that $f(\lambda)=2$ or $f(\lambda)=-2$. Thus $f^{\prime}(\lambda)=0$ and $f(\lambda)=2$ or -2 if and only if $\lambda$ is a multiple eigenvalue of (1.1) and (1.2) with $\alpha=0$ or $\alpha=\pi$.

Further, for every fixed $\lambda$ with $f(\lambda)=2$ or -2 , not indicating $\lambda$ explicitly, (3.33) implies that

$$
\begin{aligned}
& \left(k_{12} \Delta \psi_{N-1}-k_{22} \psi_{N-1}\right)\left(\left(k_{11}-k_{12}\right) \Delta \varphi_{N-1}-\left(k_{21}-k_{22}\right) \varphi_{N-1}\right) \\
& \quad=\frac{\left(k_{22} \varphi_{N-1}-\left(k_{11}-k_{12}\right) \Delta \psi_{N-1}+\left(k_{21}-k_{22}\right) \psi_{N-1}-k_{12} \Delta \varphi_{N-1}\right)^{2}}{4} .
\end{aligned}
$$

Therefore, from (3.37) and by the definition of $\delta_{j}$, we have

$$
\begin{aligned}
\delta_{j}= & \left(k_{12} \Delta \psi_{N-1}-k_{22} \psi_{N-1}\right) \\
& \cdot\left(\varphi_{j}+\frac{k_{22} \varphi_{N-1}-\left(k_{11}-k_{12}\right) \Delta \psi_{N-1}+\left(k_{21}-k_{22}\right) \psi_{N-1}-k_{12} \Delta \varphi_{N-1}}{2\left(k_{12} \Delta \psi_{N-1}-k_{22} \psi_{N-1}\right)} \psi_{j}\right)^{2}
\end{aligned}
$$

and consequently, not indicating $\lambda$ explicitly, we have

$$
\begin{aligned}
f^{\prime}= & \left(k_{12} \Delta \psi_{N-1}-k_{22} \psi_{N-1}\right) \\
& \cdot \sum_{j=0}^{N-1} w_{j}\left(\varphi_{j}+\frac{k_{22} \varphi_{N-1}-\left(k_{11}-k_{12}\right) \Delta \psi_{N-1}+\left(k_{21}-k_{22}\right) \psi_{N-1}-k_{12} \Delta \varphi_{N-1}}{2\left(k_{12} \Delta \psi_{N-1}-k_{22} \psi_{N-1}\right)} \psi_{j}\right)^{2}
\end{aligned}
$$

for every fixed $\lambda$ with $f(\lambda)=2$ or -2 . 


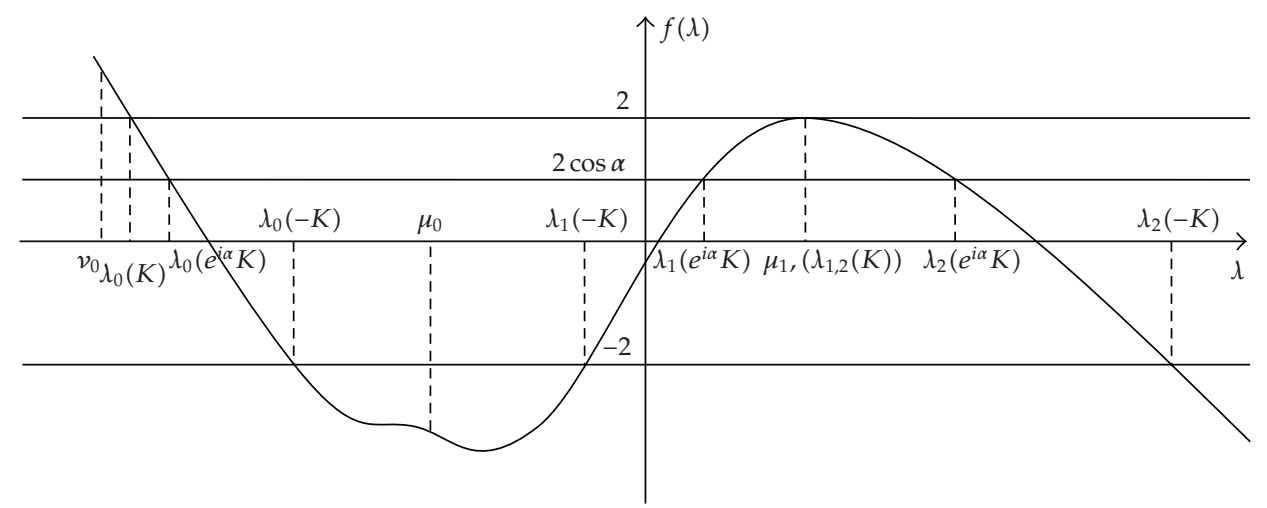

Figure 1: The graph of $f(\lambda)$.

Suppose that $f(\lambda)=2$ or -2 for some $\lambda \neq \mu_{k}\left(0 \leq k \leq N_{s}\right)$, we have $k_{12} \Delta \psi_{N-1}(\lambda)-$ $k_{22} \psi_{N-1}(\lambda) \neq 0$. From the above discussions again, $\lambda$ is a simple eigenvalue of (1.1) and (1.2) with $\alpha=0$ or $\alpha=\pi$, and $\delta_{j}$ is not identically zero for $0 \leq j \leq N-1$.

For this $\lambda \neq \mu_{k}\left(0 \leq k \leq N_{s}\right)$, (3.39) implies that $f^{\prime}(\lambda) \neq 0$, and from Proposition 3.5 (i), (ii) that $f\left(\mu_{0}\right) \leq-2, f\left(v_{0}\right) \geq 2$. Hence, $f_{\prime}^{\prime}(\lambda)<0$, where $v_{0}<\lambda<\mu_{0}$. It follows from Proposition 3.5 (i) that $f\left(\mu_{k}\right) f\left(\mu_{k+1}\right) \leq-4$ and $(-1)^{k} f \prime(\lambda)>0$, where $\mu_{k}<\lambda<\mu_{k+1}(0 \leq k \leq$ $N-3)$. By Proposition 3.5 (i), (iii), $f\left(\mu_{N-2}\right) \geq 2$ and there exists $\mu_{N-2}<\xi_{0}$ such that $f\left(\xi_{0}\right) \leq-2$ if $N$ is odd, and $f\left(\mu_{N-2}\right) \leq-2$ and there exists $\mu_{N-2}<\eta_{0}$ such that $f\left(\eta_{0}\right) \geq 2$ if $N$ is even. Hence, $(-1)^{N-2} f \prime(\lambda)>0$ where $\mu_{N-2}<\lambda$. This completes the proof.

Proposition 3.7. For any fixed $\alpha \neq 0,-\pi<\alpha<\pi$, each eigenvalue of (1.1) and (1.2) is simple.

Proof. Fix $\alpha,-\pi<\alpha<\pi$ with $\alpha \neq 0$. Suppose that $\lambda$ is an eigenvalue of the problem (1.1) and (1.2). By Proposition 3.3, we have $f^{2}(\lambda)=4 \cos ^{2} \alpha<4$. It follows from (3.33) that $\operatorname{det} I(\lambda)>0$ and the matrix $I(\lambda)$ is positive definite or negative definite. Hence, $\delta_{j}>0$ for $0 \leq j \leq N-1$ or $\delta_{j}<0$ for $0 \leq j \leq N-1$ since $\varphi_{n}$ and $\psi_{n}$ are linearly independent.

If $\lambda$ is a multiple eigenvalue of problem (1.1) and (1.2), then (3.5) holds by Proposition 3.3. By using (3.5), it can be easily verified that (3.34) holds, that is, all the entries of the matrix $I(\lambda)$ are zero. Then $\delta_{j}=0$ for $0 \leq j \leq N-1$, which is contrary to $\delta_{j} \neq 0$ for $0 \leq j \leq N-1$. Hence, $\lambda$ is a simple eigenvalue of (1.1) and (1.2). This completes the proof.

Proposition 3.8. Assume that $k_{11}>0, k_{12} \leq 0$ or $k_{11} \geq 0, k_{12}<0$. If $k$ is odd, $f\left(\mu_{k}\right)=2$, and $f^{\prime}\left(\mu_{k}\right)=0$, then $f^{\prime \prime}\left(\mu_{k}\right)<0$; if $k$ is even, $f\left(\mu_{k}\right)=-2$, and $f^{\prime}\left(\mu_{k}\right)=0$, then $f^{\prime \prime}\left(\mu_{k}\right)>0$ for $0 \leq k \leq N-2$.

Proof. We first prove the first result. Suppose that $k$ is odd, $f\left(\mu_{k}\right)=2$, and $f \prime\left(\mu_{k}\right)=0$. Then $\mu_{k}$ is a multiple eigenvalue of (1.1) and (1.2) with $\alpha=0$ by Proposition 3.6. Then by Proposition 3.3, (3.5) holds for $\lambda=\mu_{k}$ and $\alpha=0$, that is,

$$
\begin{array}{cc}
\varphi_{N-1}\left(\mu_{k}\right)=k_{11}-k_{12}, & \Delta \varphi_{N-1}\left(\mu_{k}\right)=k_{21}-k_{22}, \\
\psi_{N-1}\left(\mu_{k}\right)=k_{12}, & \Delta \psi_{N-1}\left(\mu_{k}\right)=k_{22} .
\end{array}
$$


Differentiating $f(\lambda)$ with respect to $\lambda$ two times, we get

$$
f^{\prime \prime}\left(\mu_{k}\right)=k_{22} \varphi_{N-1}^{\prime \prime}\left(\mu_{k}\right)+\left(k_{11}-k_{12}\right) \Delta \psi_{N-1}^{\prime \prime}\left(\mu_{k}\right)-\left(k_{21}-k_{22}\right) \psi_{N-1}^{\prime \prime}\left(\mu_{k}\right)-k_{12} \Delta \varphi_{N-1}^{\prime \prime}\left(\mu_{k}\right) .
$$

Differentiating (2.14) with respect to $\lambda$ two times and from (3.40), we get

$$
\begin{aligned}
& -\left(k_{22} \varphi_{N-1}^{\prime \prime}\left(\mu_{k}\right)+\left(k_{11}-k_{12}\right) \Delta \psi_{N-1}^{\prime \prime}\left(\mu_{k}\right)-\left(k_{21}-k_{22}\right) \psi_{N-1}^{\prime \prime}\left(\mu_{k}\right)-k_{12} \Delta \varphi_{N-1}^{\prime \prime}\left(\mu_{k}\right)\right) \\
& \quad+2\left(\varphi_{N}^{\prime}\left(\mu_{k}\right) \psi_{N-1}^{\prime}\left(\mu_{k}\right)-\varphi_{N-1}^{\prime}\left(\mu_{k}\right) \psi_{N}^{\prime}\left(\mu_{k}\right)\right)=0
\end{aligned}
$$

which, together with (3.41), implies that

$$
f^{\prime \prime}\left(\mu_{k}\right)=2\left(\varphi_{N}^{\prime}\left(\mu_{k}\right) \psi_{N-1}^{\prime}\left(\mu_{k}\right)-\varphi_{N-1}^{\prime}\left(\mu_{k}\right) \psi_{N}^{\prime}\left(\mu_{k}\right)\right) \text {. }
$$

On the other hand, it follows from (3.29) and (2.14) that, not indicating $\mu_{k}$ explicitly,

$$
\begin{aligned}
\varphi_{N}^{\prime} \psi_{N-1}^{\prime}-\varphi_{N-1}^{\prime} \psi_{N}^{\prime}= & \sum_{j=0}^{N-1} w_{j} \varphi_{j}\left(\varphi_{N} \psi_{j}-\varphi_{j} \psi_{N}\right) \sum_{j=0}^{N-2} w_{j} \psi_{j}\left(\varphi_{N-1} \psi_{j}-\varphi_{j} \psi_{N-1}\right) \\
& -\sum_{j=0}^{N-2} w_{j} \varphi_{j}\left(\varphi_{N-1} \psi_{j}-\varphi_{j} \psi_{N-1}\right) \sum_{j=0}^{N-1} w_{j} \psi_{j}\left(\varphi_{N} \psi_{j}-\varphi_{j} \psi_{N}\right) \\
= & \left(\sum_{j=0}^{N-1} w_{j} \varphi_{j} \psi_{j}\right)^{2}-\sum_{j=0}^{N-1} w_{j} \varphi_{j}^{2} \sum_{j=0}^{N-1} w_{j} \psi_{j}^{2} .
\end{aligned}
$$

Since $\varphi_{n}$ and $\psi_{n}$ are linearly independent on $[-1, N]$, the above relation implies that $f \prime \prime\left(\mu_{k}\right)<$ 0 by Hölder's inequality, which proves the first conclusion.

The second conclusion can be shown similarly. Hence, the proof is complete.

Finally, we turn to the proof of Theorem 3.1.

Proof of Theorem 3.1. By Propositions 3.3-3.8, and the intermediate value theorem, one can obtain the graph of $f$ (see Figure 1), which implies the results of Theorem 3.1. We now give its detailed proof.

By Propositions 3.3-3.6, $f\left(\mu_{0}\right) \leq-2, f^{\prime}(\lambda)<0$ for all $\lambda<\mu_{0}$ with $-2 \leq f(\lambda) \leq 2$, and there exists $v_{0}<\mu_{0}$ such that $f\left(v_{0}\right) \geq 2$. Therefore, by the continuity of $f(\lambda)$ and the intermediate value theorem, (1.1) and (1.2) with $\alpha=0$ has only one eigenvalue $\lambda_{0}(K)<\mu_{0}$, (1.1) and (1.2) with $\alpha=\pi$ has only one eigenvalue $\lambda_{0}(-K) \leq \mu_{0}$, and (1.1) and (1.2) with $\alpha \neq 0,-\pi<\alpha<\pi$ has only one eigenvalue $\lambda_{0}(K)<\lambda_{0}\left(e^{i \alpha} K\right)<\lambda_{0}(-K)$, and they satisfy

$$
\mathcal{v}_{0} \leq \lambda_{0}(K)<\lambda_{0}\left(e^{i \alpha} K\right)<\lambda_{0}(-K) \leq \mu_{0} .
$$

Similarly, by Propositions 3.3-3.6, the continuity of $f(\lambda)$, and the intermediate value theorem, $f(\lambda)$ reaches $-2,2 \cos \alpha(\alpha \neq 0,-\pi<\alpha<\pi)$, and 2 exactly one time, respectively, between 


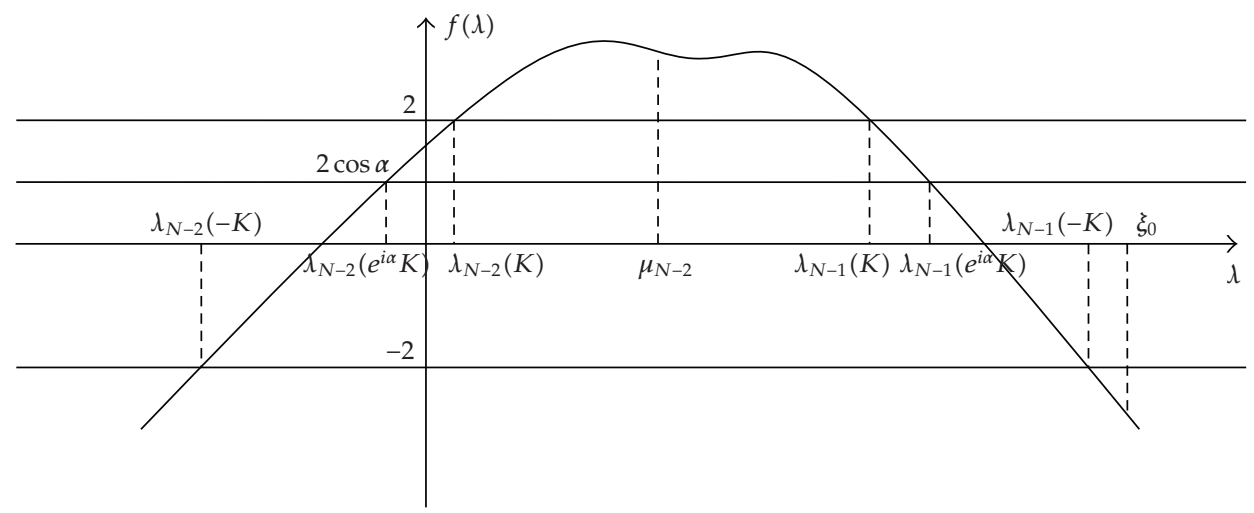

Figure 2: The graph of $f(\lambda)$ in the case that $N$ is odd.

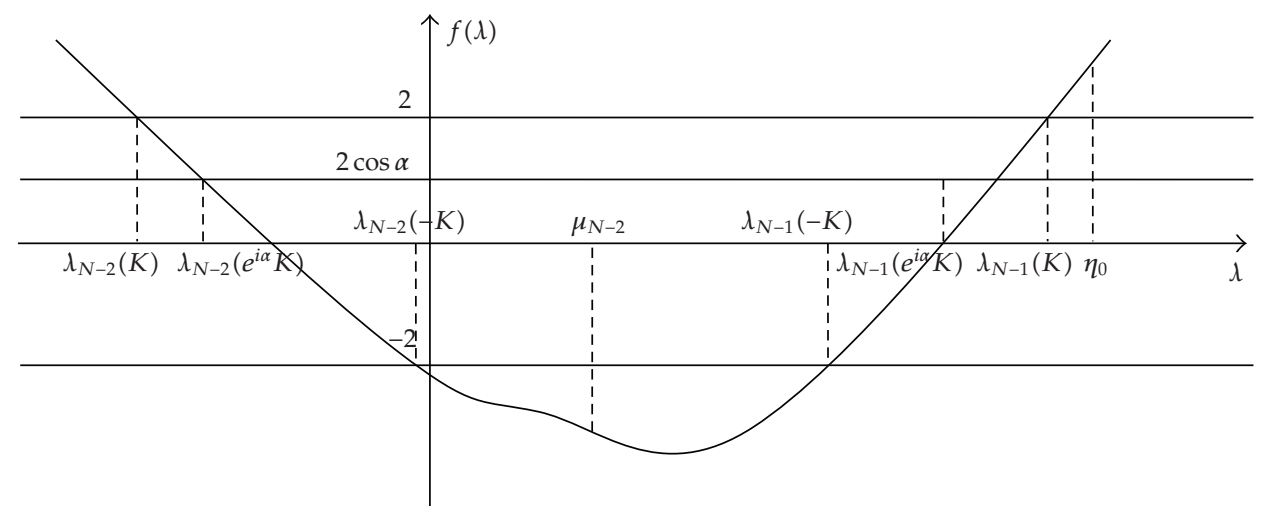

Figure 3: The graph of $f(\lambda)$ in the case that $N$ is even.

any two consecutive eigenvalues of the separated boundary value problem (1.1) with (2.7). Hence, (1.1) and (1.2) with $\alpha=0 ; \alpha \neq 0,-\pi<\alpha<\pi ; \alpha=\pi$ has only one eigenvalue between any two consecutive eigenvalues of (1.1) with (2.7), respectively. In addition, by Proposition 3.6, if $f\left(\mu_{k}\right)=2$ or -2 and $f^{\prime}\left(\mu_{k}\right)=0$, then $\mu_{k}$ is not only an eigenvalue of (1.1) with (2.7) but also a multiple eigenvalue of (1.1) and (1.2) with $\alpha=0$ and $\alpha=\pi$.

By Proposition 3.5 (i), if $N$ is odd, $f\left(\mu_{N-2}\right) \geq 2$ and if $N$ is even, $f\left(\mu_{N-2}\right) \leq-2$. It follows (3.22) that if $N$ is odd, then $f(\lambda) \rightarrow-\infty$ as $\lambda \rightarrow+\infty$, and if $N$ is even, then $f(\lambda) \rightarrow$ $+\infty$ as $\lambda \rightarrow+\infty$. Hence, if $N$ is odd, then there exists a constant $\xi_{0}>\mu_{N-2}$ such that $f\left(\xi_{0}\right) \leq-2$, which, together with Proposition 3.6, implies that (1.1) and (1.2) with $\alpha=0 ; \alpha \neq 0,-\pi<\alpha<\pi$; $\alpha=\pi$, has only one eigenvalue $\lambda_{N-1}(K), \lambda_{N-1}\left(e^{i \alpha} K\right)$, and $\lambda_{N-1}(-K)$, satisfying

$$
\mu_{N-2} \leq \lambda_{N-1}(K)<\lambda_{N-1}\left(e^{i \alpha} K\right)<\lambda_{N-1}(-K) \leq \xi_{0}
$$

(see Figure 2). Similarly, in the other case that $N$ is even, there exists a constant $\eta_{0}>\mu_{N-2}$ such that $f\left(\eta_{0}\right) \geq 2$, which, together with Proposition 3.6, implies that (1.1) and (1.2) with 
$\alpha=0 ; \alpha \neq 0,-\pi<\alpha<\pi ; \alpha=\pi$ has only one eigenvalue $\lambda_{N-1}(K), \lambda_{N-1}\left(e^{i \alpha} K\right)$, and $\lambda_{N-1}(-K)$, satisfying

$$
\mu_{N-2} \leq \lambda_{N-1}(-K)<\lambda_{N-1}\left(e^{i \alpha} K\right)<\lambda_{N-1}(K) \leq \eta_{0}
$$

(see Figure 3). Therefore, we get that (1.1) and (1.2) with $\alpha \neq 0,-\pi<\alpha<\pi$, has $N$ eigenvalues and it is real and satisfies

$$
\begin{aligned}
v_{0} & \leq \lambda_{0}(K)<\lambda_{0}\left(e^{i \alpha} K\right)<\lambda_{0}(-K) \leq \mu_{0} \leq \lambda_{1}(-K)<\lambda_{1}\left(e^{i \alpha} K\right)<\lambda_{1}(K) \leq \mu_{1} \\
& \leq \lambda_{2}(K)<\lambda_{2}\left(e^{i \alpha} K\right)<\lambda_{2}(-K) \leq \mu_{2} \leq \lambda_{3}(-K)<\lambda_{3}\left(e^{i \alpha} K\right)<\lambda_{3}(K) \leq \mu_{3} \\
& \leq \cdots \leq \mu_{N-3} \leq \lambda_{N-2}(-K)<\lambda_{N-2}\left(e^{i \alpha} K\right)<\lambda_{N-2}(K) \leq \mu_{N-2} \leq \lambda_{N-1}(K) \\
& <\lambda_{N-1}\left(e^{i \alpha} K\right)<\lambda_{N-1}(-K) \leq \xi_{0}, \quad \text { if } N \text { is odd, } \\
v_{0} & \leq \lambda_{0}(K)<\lambda_{0}\left(e^{i \alpha} K\right)<\lambda_{0}(-K) \leq \mu_{0} \leq \lambda_{1}(-K)<\lambda_{1}\left(e^{i \alpha} K\right)<\lambda_{1}(K) \leq \mu_{1} \\
& \leq \lambda_{2}(K)<\lambda_{2}\left(e^{i \alpha} K\right)<\lambda_{2}(-K) \leq \mu_{2} \leq \lambda_{3}(-K)<\lambda_{3}\left(e^{i \alpha} K\right)<\lambda_{3}(K) \leq \mu_{3} \\
& \leq \cdots \leq \mu_{N-3} \leq \lambda_{N-2}(K)<\lambda_{N-2}\left(e^{i \alpha} K\right)<\lambda_{N-2}(-K) \leq \mu_{N-2} \leq \lambda_{N-1}(-K) \\
& <\lambda_{N-1}\left(e^{i \alpha} K\right)<\lambda_{N-1}(K) \leq \eta_{0}, \quad \text { if } N \text { is even. }
\end{aligned}
$$

This completes the proof.

Remark 3.9. Let $K=I$, that is, $k_{11}=k_{22}=1, k_{12}=k_{21}=0$. Then $f(\lambda)=\varphi_{N-1}(\lambda)+\psi_{N}(\lambda)$. In this case, Propositions 3.5 and 3.8 are the same as those mentioned in [4, Propositions 3.1, 3.3-3.5], respectively, and most of the results of Proposition 3.6 are the same as the results of [4, Proposition 3.2].

\section{Acknowledgments}

Many thanks to Johnny Henderson (the editor) and the anonymous reviewers for helpful comments and suggestions. This research was supported by the Natural Scientific Foundation of Shandong Province (Grant Y2007A27), (Grant Y2008A28), and the Fund of Doctoral Program Research of University of Jinan (B0621).

\section{References}

[1] F. V. Atkinson, Discrete and Continuous Boundary Problems, vol. 8 of Mathematics in Science and Engineering, Academic Press, New York, NY, USA, 1964.

[2] Y. Shi and S. Chen, "Spectral theory of second-order vector difference equations," Journal of Mathematical Analysis and Applications, vol. 239, no. 2, pp. 195-212, 1999.

[3] R. P. Agarwal and P. J. Y. Wong, Advanced Topics in Difference Equations, vol. 404 of Mathematics and Its Applications, Kluwer Academic Publishers, Dordrecht, The Netherlands, 1997. 
[4] Y. Wang and Y. Shi, "Eigenvalues of second-order difference equations with periodic and antiperiodic boundary conditions," Journal of Mathematical Analysis and Applications, vol. 309, no. 1, pp. 56-69, 2005.

[5] E. A. Coddington and N. Levinson, Theory of Ordinary Differential Equations, McGraw-Hill, New York, NY, USA, 1955.

[6] J. K. Hale, Ordinary Differential Equations, vol. 20 of Pure and Applied Mathematics, Wiley-Interscience, New York, NY, USA, 1969.

[7] W. Magnus and S. Winkler, Hill's Equation, Interscience Tracts in Pure and Applied Mathematics, no. 20, Wiley-Interscience, New York, NY, USA, 1966.

[8] M. Zhang, "The rotation number approach to eigenvalues of the one-dimensional $p$-Laplacian with periodic potentials," Journal of the London Mathematical Society, vol. 64, no. 1, pp. 125-143, 2001.

[9] R. P. Agarwal, M. Bohner, and P. J. Y. Wong, "Sturm-Liouville eigenvalue problems on time scales," Applied Mathematics and Computation, vol. 99, no. 2-3, pp. 153-166, 1999. 